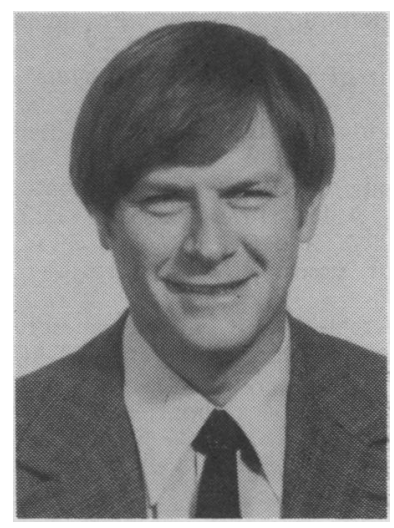

\section{OROFACIAL INJURIES AND THE USE OF MOUTHGUARDS BY THE 1984 GREAT BRITAIN RUGBY LEAGUE TOURING TEAM}

\author{
P. J. CHAPMAN, MB, BS, MDSc
}

Dept. of Oral Surgery, University of Queensland Dental School, Turbot Street, Brisbane, Queensland 4000, Australia

P. J. Chapman

\title{
ABSTRACT
}

Mouthguards are considered by most authorities to be an essential part of equipment for players in any body-contact sports, especially the combative type e.g. Rugby Union, Rugby League, American Football (gridiron), boxing, etc. (Turner, 1977). The qualities provided by the mouthguard are dental protection, especially of the upper anterior teeth, soft tissue protection around the mouth, a reduction in the risk of fracture of the mandible, and a reduction in the concussion force from a blow to the mandible (Clegg, 1969; Upson, 1982; Davies et al, 1977). Of the 28 players interviewed, only 7 (25\%) wore mouthguards, the commonest reason for not using a mouthguard being difficulty with breathing, a finding in common with other similar surveys (Davies et al, 1977). In view of the fact that $17(60.7 \%)$ had sustained oral injuries - dental and jaw injuries, intra-oral and circumoral lacerations, in the past, it was a surprising response to find that only $2(7.2 \%)$ stated that mouthguards should be compulsory when playing Rugby League football.

Key words: Mouthguard usage, Dental injuries, International level, Rugby League.

\section{INTRODUCTION}

Mouthguards are perhaps the commonest form of protective device now worn in contact sport. However, this has not always been the case. Up to 25 years ago there was very little consideration given to the protection of the mouth, the jaws and teeth. Since the early 1960's, studies particularly in the United States of America have shown the advantages of protection provided when wearing mouthguards with up to a 90 per cent reduction in dental injuries when using a properly fitted mouthguard (Davies et al, 1977; Hughston, 1980). The cost effectiveness of these devices is very great as dental injuries can be very costly. When related to the cost of a mouthguard, this benefit is easily seen (Heintz, 1979).

The opportunity was taken to assess the attitude to and the usage of mouthguards by the international team touring Australia in 1984. A similar study of the 1984 Australian Rugby League team was carried out and showed that $80 \%$ of players wore mouthguards. It is important to assess the results in light of four points. Firstly the overall usage by such players, since these sportsmen have a high public profile, especially on television, and what they are seen to do (wear mouthguards or not) or say (attitude to mouthguards) has a marked influence on younger footballers who follow that particular code. Secondly, such figures reflect the result of dental public education programmes, aimed at promoting mouthguard usage in contact sports. Thirdly, it is also a reflection on an aspect of preventive sports medicine which may need to be emphasised by team doctors to players, especially in view of the known reduction in concussion force from a blow to the mandible when a mouthguard is worn. Finally, the overall cost of expensive treatment for largely preventable dental injuries would be almost entirely eliminated.

\section{RESULTS}

There were twenty-eight players who answered the questionnaire. The average length of playing Rugby League football was 12.5 years. Their average age was 24.1 years. Of the total, all players agreed that a custom mouthguard could reduce injuries to the teeth and around the mouth. However, 26
(92.8\%) stated that wearing mouthguards in Rugby League football should not be made compulsory and should be left to the individual choice. Those 2 players who believed in compulsory wearing of mouthguards wore mouthguards themselves.

Seventeen $(60.7 \%)$ had suffered dental or intra-oral injuries in the past: this was usually caused by accidental contact with a knee or elbow, less commonly by contact with a boot or a head. At the time of injury, only 1 of the 17 (5.8\%) was wearing a mouthguard - this was a custom mouthguard and the injury was to a lower front tooth, a type of accident that is still possible even when wearing a mouthguard. Of the injuries, 14 of the $17(82.3 \%)$ required dental treatment the others were lacerations to the upper lip ( 2 cases) and tongue (1 case), all required suturing.

Seven of the players $(25 \%)$ used a mouthguard regularly. Of the $75 \%$ who did not, $9(42.8 \%)$ had been advised to wear a mouthguard, mainly by the team doctor or a dentist. Also, of the 21 who did not wear a mouthguard, 6 (28.5\%) had previously tried but since discarded wearing a mouthguard, of which 5 were custom mouthguards. Their complaint with the custom mouthguard was difficulty in breathing in 3 cases, difficulty in breathing and nausea in 1 case, and difficulty in speaking in 1 case. In the case of the other mouthguard tried (a stock mouthguard), the fitting was bad and it cut into the mouth when worn.

Regarding those who wore mouthguards, all of which were custom mouthguards, their age range was 19 to 29 years, (average 24 years) and the average playing period was 13.4 years. Five of the seven stated they had previously received a dental injury when playing. Only 1 of the 5 was wearing a mouthguard (a custom mouthguard) at that time and he had a lower tooth avulsed, as mentioned previously. The other 4 had sustained avulsed or fractured upper anterior teeth. Each of these four players started wearing a mouthguard after the injury, as a direct result of the injury. The average age at commencement of wearing a mouthguard was 20.2 years and the average number of years after they started playing the 
sport to when they began to wear a mouthguard was 9.7 years. Finally, three said they would be prepared to play a match without a mouthguard, one said he definitely would not, and the remaining three said they would play without a mouth. guard only if absolutely required to.

\section{DISCUSSION}

Historically, the first sport in which mouthguards were used was boxing and in 1913 there were reports of boxers in England using some form of crude mouthguard (Wehner and Henderson, 1965). Boxers needed something to protect them from the violent blows they received in their sport; blows that lacerated lips, fractured teeth and bone, and that sometimes resulted in head injury and death. These early mouthguards were curved trays of rubber-type materials which were worn on the upper teeth. They had no retention and were held in place by gritting the teeth together, but did provide some degree of dental protection. More importantly, the boxing fraternity showed an early understanding that impacts delivered to the lower jaw were transmitted to the brain, often with dramatic results, and their efforts to minimise this was one of the first significant developments in protective equipment in sports (Hughston, 1980).

Research was later commenced on injury protection of this region and most of this was done in USA in gridiron football, and results were published in the dental literature in the 1950's and 1960's (Heintz, 1968). These results were confirmed by other studies in different countries involving various contact sports and showed the value of mouthguard protection (Clegg, 1969).

In the various football codes and other popular contact sports, injury to the head and facial region is caused largely by the following mechanism (Wood, 1972).

1. Direct impact from in front (as in a straight punch) that can cause fracture or damage to the anterior teeth (especially the upper), fracture of the mandible and lacerations to the upper or lower lips.

2. Impact to the mandible from below (as in an uppercut) that cause fracture or damage to the teeth of either jaw (as the teeth suddenly crash together), fracture of the mandible and lacerations of the lips or tongue.

3. Either of the previously mentioned types of impact also can cause brain injury, (concussion, cerebral contusions and even death) by direct transmission of the force to the skull and brain, mainly through the mandibular condyles.

The mouthguard provides protection against such cerebral injury because the condyles move forward in the glenoid fossa when a mouthguard is worn. This is easily demonstrated by having a player first close his teeth together. Ask him to palpate the condyles on each. side. Then have him insert his mouthguard, and close his teeth together again. Forward movement of the condyles will be noticed. Therefore, following impact to the mandible, the transmission of forces through the condyle to the skull and the brain is reduced, reducing the possibility of concussion and brain injury. This concept has been confirmed by research (Hickey et al, 1967). There is also a reduction of transmission of impact forces to the cervical spine by this condyle movement, with a lessening risk of cervical spine injury (Hayward, 1978; Stenger et al, 1964).

These blows are usually from fists, elbows, or knees and may be accidental and illegal or deliberate and legal, depending on the sport involved. The functions of a mouthguard must encompass these possibilities. However, since the mouthguard is usually worn on the maxillary teeth, being more prominent and therefore exposed to injury, most protection is provided for the upper anterior teeth, and there is still a risk of injury to the lower teeth when wearing a custom type mouthguard.
The shock absorption quality of mouthguards is a very important concept in the prevention of injuries, and is the reason that resilient type materials are used.

When the above points are made known to a player involved in any of the contact sports, there should be a psychological enhancement of his attitude and consequently ability in actual competition when wearing a mouthguard (Davies et al, 1977).

The ideal mouthguard should have the following qualities (Turner, 1977):

Provide the functions as outlined above.

Be comfortable.

Good retention - unlikely to be dislodged in sudden impact. This would cause possible airway problems if a player is rendered unconscious.

No interference with speech or breathing.

Be durable and able to last for 1 or 2 seasons.

Be affordable.

Be odourless and tasteless and non-irritating to the oral tissues.

The custom type conforms best to the ideal qualities of a mouthguard (Turner, 1977). They are made of vinyl plastic which is individually moulded on a model of a person's upper teeth after impressions have been taken, and are constructed by a dentist. The cost of a custom type mouthguard is far more than the other two types, stock ("gum shields") and mouth-formed mouthguards: however the advantages in real terms outweigh any cost disadvantage. Custom-made mouthguards are therefore recommended for all types of contact sports (Heintz, 1979).

Some players may be tempted, because of the cost of a custom mouthguard, to purchase a stock type (Upson, 1982). This type is kept in place by biting the teeth together. A near fatal accident occurred to a Rugby Union player wearing such a type, which was dislodged during play, occluding the airway and causing respiratory distress. Fortunately, a medical trained spectator quickly realising the problem removed the mouthguard and resuscitated the player (Turner, 1977). This is the main reason why these sort of mouthguards are dangerous and should not be allowed to be sold (Turner, 1977). It would be highly unlikely that a custom mouthguard would be dislodged in similar situations, but it is always a possibility with stock mouthguards.

The age when mouthguards should be first worn is when children first play organised contact sport at school, usually by about 10 to 12 years. Initially dental injuries in organised contact sport dt school only cause about 15 per cent of dental injuries to 13 years of age but by adolescence they account for over 50 per cent of dental injuries (Nicholas, 1980; Lees and Gaskell, 1976). Children should therefore be encouraged to use mouthguards early (Davies et al, 1977; de Wet, 1981). Also it is far more likely that they will use them later in life if they have used them at an earlier age (Heintz, 1979).

Many players who wear mouthguards during sport do not wear them during training. This is a problem because dental injuries can also occur in training as well as in an actual game. Sugarman (1983) has estimated the risk of injury during actual competition to the risk during training in Rugby Union is about 4.5 to 1 . This would similarly apply to Rugby League. Also players will not become really familiar with the feel and fit of the mouthguard and its effect on speech and breathing if not worn during training. Consequently it may feel awkward when worn during a game, and may impair the player's concentration. It is therefore important that mouthguards are worn during training as well as during actual competition (Heintz, 1968). 
It was surprising to find that only a quarter of the international football team used mouthguards. The reason usually given was difficulty in breathing. It has been shown that mouthguards do significantly impair the oral airflow, in a limited study (Luke et al, 1982). This is due to the thickness of the mouthguard $(2.3 \mathrm{~mm})$ effectively reducing the dimensions of the oral airway between the upper and lower incisor teeth, by the same amount $(2.3 \mathrm{~mm})$ and increasing resistance to airflow. The normal distance between upper and lower incisor teeth in a person lightly breathing during mild exertion is only about 2 to $3 \mathrm{~mm}$, and when breathing heavily through the mouth, such as during moderate to heavy physical exertion, the distance is about $1 \mathrm{~cm}$. Thus, in a situation when a mouthguard is being worn, this interincisal distance is virtually obliterated during mild exertion and reduced to $7-8 \mathrm{~mm}$ during moderate to heavy exertion. Therefore a mouthguard would have most influence on breathing ability under conditions of mild exertion. In conditions of moderate to heavy exertion the effect would be less but there is still a theoretical reduction in the air flow dimension of about $30 \%$. This was confirmed in the experiments by Luke et al (1982). The practical evidence of mouthguards causing airway interference is the common occurrence of footballers taking their mouthguards out after an exertion and whilst recovering.

Of course the position of the upper lip can also effectively reduce the oral airway dimensions. In more heavy type exertion, the upper lip is usually retracted purposely to prevent any interference with the oral airflow, whereas in lesser type exertion the lip usually is not retracted, and the edge lies just below the edge of the upper incisors, by about $2.3 \mathrm{~mm}$. If a mouthguard is worn, because the upper lip is distended and stretched by the bulk of the mouthguard, lip interference to oral airflow is eliminated.

The nausea mentioned by one person could be eliminated by careful trimming of the posterior edge of the mouthguard by a dentist and the problem with speech mentioned by another is overcome by using the mouthguard at training. An old coaching adage is "what you play in, you train in", and this applies especially to mouthguards. It is interesting to note that the author fabricated a mouthguard for Brian Noble, the touring captain, and he wore it one week later in the Third Test against Australia. He had never worn a mouthguard before and after using it at a few training runs, was confident at wearing it for the Test. Although he had lost the four upper front teeth previously, he stated that he frequently sustained deep cuts to the inner aspect of the upper lip during a match by impingement of the upper lip on the upper canine teeth.

Clegg (1969) reported that of 100 Rugby League club players a third had sustained orofacial injuries. Upson (1982) reported that of 100 Rugby Union club players, $27 \%$ had sustained previous dental damage. In this present survey, by com: parison, almost two thirds of the team had suffered orofacial injuries. This may reflect the suggestion that the greater the grade of competition, the higher the risk of injuries occurring in footballers, with the highest incidence in representative matches (Myers, 1980). Although that survey was done in Rugby Union, the results would be equally applicable to Rugby League. Clegg (1969) also reported that there was a complete elimination of dental type injuries after players started wearing custom mouthquards, the incidence of fractures of the mandible was also reduced, as was the rate of concussion. Upson (1982) has reported that whilst $66 \%$ had worn a mouthguard at some stage, only $38 \%$ still wore a mouthguard, and only a quarter of these were custom type. Dennis and Parker (1972) had previously reported that $60 \%$ of all mouthguards were discarded for various reasons, the players being content to accept the increased risk of personal injury. The highest rate of discontinuance is with the stock type, over $60 \%$.

Finally, the most important aspect of mouthguard protection is the concussion saving effects and this alone should make the wearing of mouthguards compulsory. However a controversial aspect also to be considered by authorities of all codes is that if wearing mouthguards was made compulsory, it would eliminate claims of biting in the sport. There seems very solid argument for making mouthguards compulsory in contact sports, instead of leaving it to individual preference (de Wet, 1981). Since 1973, all high school and college football players in the USA have been required to wear mouthguards (Picozzi, 1975). Although wearing mouthguards is not mandatory at the professional level of the sport, most professional players wear mouthguards because they have become accustomed to wearing them at high school and college (Heintz, 1979).

\section{ACKNOWLEDGEMENTS}

The author wishes to express sincere thanks to Brian Noble, the other team members and Frank Myler (Coach) for their co-operation.

\section{References}

Clegg, J. H., 1969 "Mouth protection for the rugby football player". Brit.Dent.J. 127: 341-343

Davies, R. M., Bradley, D., Hale, R. W., Laird, W. R. E. and Thomas, P. D., 1977 "The prevalence of dental injuries in rugby players and their attitude to mouthguards". B.J.Sports Med. 11: 72-74.

Dennis, C. G. and Parker, D. A. S., 1972 "Mouthguards in Australian sport". Aust.Dent.J. 17: 228-235. i.

De Wet, F. A., 1981 "The prevention of orofacial sports injuries in the adolescent"' Int.Dent.J. 31: 313-319.

Hay wood, J. R., 1978 "Recent advances in the management of facial fractures". Int.J.Oral Surg. 7: 263-264.

Heintz, W. D., 1968 "Mouth protectors: a progress report". JADA 77: $632-636$

Heintz, W. D., 1979 "Mouth protectors in sports". Phys.Sports Med. 7: $45-46$.

Hickey, J. C., Morris, A. L., Carlson, L. D. and Seward, T. E., 1967 "Relation of mouth protectors to cranial pressure and deformation". JADA 74: 735-740.

Hughston, J. C., 1980 "Prevention of dental injuries in sports". Amer. J.Sports Med. 8: 61-62.

Lees, G. H. and Gaskell, P. H. F., 1976 "Injuries to the mouth and teeth in an undergraduate population". Brit.Dent.J. 140: 107. 108.

Luke, R. Taylor, G. and Kaplin, R. 1982 "The effect of a mouthguard on airflow". Diastema 10: 56-57.

Myers, P. T., 1980 "Injuries presenting from Rugby Union football". Med.J.Aust. 2: 17-20.

Nicholas, N. K., 1980 "Dental injuries in primary and intermediate school children". N.Z.Dent.J. 76: 8-11.

Picozzi, A., 1975 "Mouth protectors". Dent.Clin.N.Amer. 19: 385-388.

Stenger, J. M., Lawson, E. A., Wright, J. M. and Ricketts, J., 1964 "Mouthguards - protection against shock to head, neck and teeth". JADA 69: 273-281.

Sugarman, S., 1983 "Injuries in an Australian schools Rugby Union season". Aust.J.Sports Med.and Ex.Sci. 15: 5-14.

Turner, C. H., 1977 "Mouth protectors". Brit.Dent.J. 143: 82-86.

Upson, N., 1982 "Dental injuries and attitudes of rugby players to mouthguards". Brit.J.Sports Med. 16 (4): 241-244.

Wehner, P. J. and Henderson, D., 1965 "Maximum prevention and preservation". Dent.Clin.N.Amer. 9: 493-498.

Wood, A. W. S., 1972 "Head protection". Oral Health 62: 23-33. 